\title{
Development of high-temperature electrochemical TEM and its application on solid oxide electrolysis cells
}

SørenBredmose Simonsen, Waynah Lou Dacayan, Zhongtao Ma, ChristodoulosChatzichristodoulou, Wenjing Zhang and Kristian Speranza Mølhave

\section{Technical University of Denmark, United States}

We are transforming a transmission electron microscope (TEM) into a generic high-temperature electrochemical workstation for solid oxide electrolysis and fuel cell (SOEC/SOFC) materials. We focus on the combination of TEM and electrochemical impedance spectroscopy (EIS). EIS is today one of the most powerful characterization methods for distinguishing electrochemical resistances for different processes (electronic conductivity, ionic conductivity, gas diffusion, catalytic reactions etc.) [1].

SOEC and SOFC are a promising technologies for efficient green energy storage by conversion of electrical energy to chemical energy by SOEC and back to electrical energy by SOFC [2]. SOECs and SOFCs operate in reactive gasses $\left(\mathrm{O}_{2}, \mathrm{H}_{2} \mathrm{O}, \mathrm{CO}_{2}\right)$ at high temperatures, typically $\left.\geq 800^{\circ} \mathrm{C}\right)$, which makes it challenging to study them via in situ TEM. In addition, SOECs are composed of hard and brittle ceramics and can be difficult to thin for electron transparency and manipulate without breaking. However, post mortem electron microscopy does not give direct insight into time, temperature and electrical potential dependencies of cell degradation, and in situ characterization is therefore needed.

Here we present different approaches for designing high-temperature electrochemical TEM experiments. In one study, we are model SOEC/SOFCs composed of materials commonly used in state-of-the-art SOEC/SOFCs. The cells are prepared by pulsed laser deposition (PLD). In another study the samples are electrospun gadolinia-doped ceria (CGO) nanofibers representing electrode and electrolyte materials in an SOEC/SOFC.

For the experiments, an ETEM is used in combination with custom-made and commercial heating/biasing TEM holders and a potentiostat and impedance analyzer for measuring I-V response as well as EIS data. Observations include segregation and grain formation, accelerate as a function of temperature, and is promoted by the presence of $\mathrm{O}_{2}$. Structural degradations are observed both as a response to heating and applied polarization.
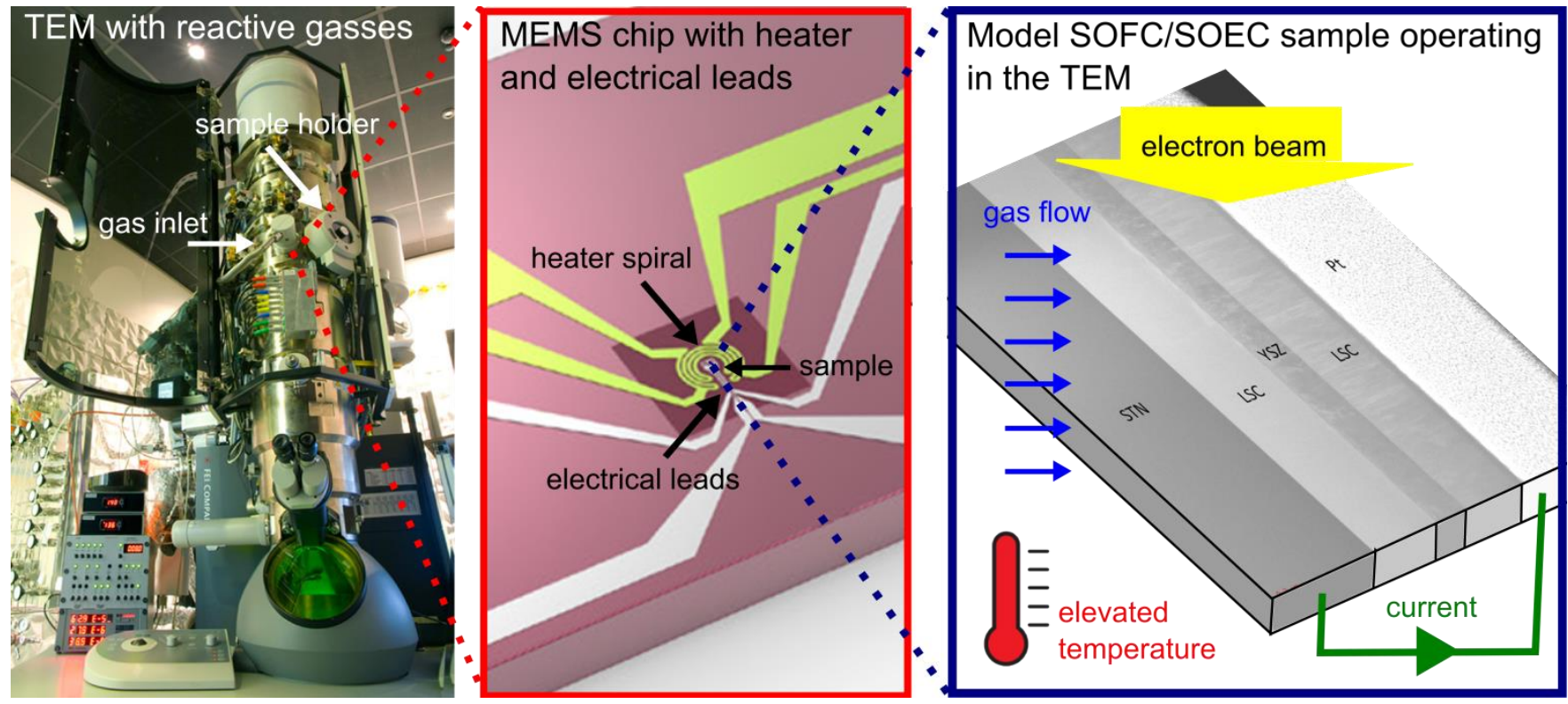

Figure 1. Fig. 1: Illustration of one approach for high-temperature electrochemical TEM 


\section{References}

1. M.J. Jørgensen, S. Primdahl, M.B. MogensenCharacterization of composite SOFC cathodes using electrochemical impedance spectroscopyElectrochimica Acta, 44 (1999) 4195-4201

2. N.Q. Mitili, M.B. Mogensen, Reversible Solid Oxide Fuel Cell Technology for Green Fuel and Power Production, Interface. 22 (2013) 55-62. 